\title{
Review
}

\section{Virotherapy clinical trials for regional disease: In situ immune modulation using recombinant poxvirus vectors}

\author{
Michael J Mastrangelo, ${ }^{1}$ and Edmund C Lattime ${ }^{2}$ \\ ${ }^{1}$ Department of Medicine, Division of Medical Oncology, Thomas Jefferson University, Philadelphia, Pennsylvania \\ 19107, USA; and ${ }^{2}$ Department of Surgery, UMDNJ/Robert Wood Johnson Medical School and The Cancer \\ Institute of New Jersey, New Brunswick, New Jersey 08901, USA.
}

\begin{abstract}
The ability of viruses to readily infect tumor cells both in vitro and in vivo has resulted in their study as antitumor agents through a variety of strategies. Replicating and conditionally replicating viruses and recombinant viruses encoding genes for toxins and/or prodrugs have been studied for their direct antitumor activity with promising results. However, to date, the lack of a targettable construct able to localize to all tumors following systemic administration has proven to be a major limitation in their use for metastatic disease. The ability of a variety of well - characterized viruses to serve as vectors for expression of tumor antigens and/or cytokines has also resulted in their study as immunotherapeutic agents. In this review, we discuss preclinical and clinical data that support the use of recombinant poxviruses as vectors for in situ tumor transfection with immune-enhancing cytokines and immune costimulatory antigens. We hypothesize that such an approach will ultimately lead to enhanced immune recognition of tumor and the development of an effective systemic antitumor immune response capable of eradicating primary and metastatic tumor foci. Cancer Gene Therapy (2002) 9, 1013-1021 doi:10.1038/sj.cgt.7700538
\end{abstract}

Keywords: vaccinia; immunotherapy; clinical trials; vaccine; melanoma; bladder cancer

A s reviewed in the contributions to this volume, studies from a variety of laboratories, each with its own particular focus, have used viruses and viral vectors as antitumor agents (reviewed in Ref. [1]). These approaches range from direct tumor lysis by replicating or conditionally replicating viruses, to the use of viral vectors to carry toxins or prodrugs to tumor, to the use of viral vectors to, in one way or another, induce antitumor immunity as discussed in this review. Each approach has its own strengths and limitations and it is likely, as has been the long-held conclusion from traditional anticancer therapies such as surgery, chemotherapy, and radiation, that no single modality will be the "magic bullet," but rather that combinations of modalities will likely be required for effective tumor eradication. Regardless of the strategy used, for an approach to be effective, it will be necessary to eliminate both identifiable tumor deposits as well as micrometastatic disease. For this reason, immunotherapy strategies, which hold out the promise of developing systemic antitumor effects, have held particular interest. To date, given the inability to target viral vectors administered systemically to all tumor, immunotherapy-based strategies continue to represent a major focus of clinical virotherapy trials.

Received September 16, 2002.

Address correspondence and reprint requests to: Dr Edmund C Lattime, The Cancer Institute of New Jersey, 195 Little Albany Street, New Brunswick, NJ 08901, USA.E-mail: edmund.lattime@umdnj.edu
The concept that the immune response may be manipulated so as to eliminate established neoplasms is an appealing one, which has been under study for decades. This concept stemmed from the early suggestion of Thomas ${ }^{2}$ in 1959 that the immune response might be useful in ridding the body of aberrant cells and was later refined into the immune surveillance hypothesis of Burnet ${ }^{3}$ in 1970, which, in its simplest form, hypothesized that the immune system would recognize incipient tumors as foreign and reject them and that only those tumors that evaded this surveillance mechanism would persist and grow. Early clinical support for the potential effectiveness of the immune system in eradicating tumors was based on the reports that a number of human tumors, especially melanoma and renal cell, spontaneously regress presumably by the development of an antitumor immune response. ${ }^{4,5}$ Based on these and other findings as well as a rapidly evolving understanding of basic immune regulation, studies by numerous investigators in preclinical and clinical settings have continued to focus on harnessing the immune response as a therapeutic for malignancy.

To date, investigators have primarily approached tumor immunotherapy in three ways (reviewed in Ref. [6]). Local therapy with immune-active adjuvants has been shown to be highly effective in the case of localized tumors of the skin and bladder. ${ }^{7-9}$ Tumor vaccines represent the most studied approach to therapy. In their earliest manifestations, these included the use of either whole tumor cells given unmodified or following modification with viral antigens or haptens (reviewed in Refs. [10,11]) followed by the use of tumor extracts and most recently defined protein antigens 
and peptides ${ }^{12}$ injected directly or used to pulse antigenpresenting cells, which were subsequently used as vaccines. Success in inducing regression of clinically evident disease using these vaccines has been quite limited. However, in the adjuvant setting where one would expect minimal residual disease, prolongation of disease-free survival has been reported using these first-generation approaches. More recently, gene-based strategies have come to the fore with plasmid- and viral vectors-encoding tumor antigens being used as vaccines and as vectors for delivering immuneenhancing cytokines and costimulatory molecules to tumor in situ.

When one considers the choice of viral vectors for gene therapy, candidates usually include adenovirus and retrovirus families and, in the case of retrovirus, most recently, the HIV-derived lentiviral vectors (reviewed in Ref. [1]). Poxvirus vectors are often absent from the list of candidates for most studies. Indeed, whereas a number of characteristics of the lifecycle of poxvirus infection make them poor candidates for long-term expression, they have a number of properties that make them ideal for consideration in immunotherapy applications. Most notably, they are large viruses that are routinely shown to allow the insertion of multiple genes without compromising infectivity. In addition, the poxvirus lifecycle is limited to the cytoplasm, thus eliminating any concern for integration into the genome. Finally, they have a long history as effective and safe vaccines, first used successfully in the 1700 s as a vaccine for smallpox.

While not traditionally thought of as viral or gene therapy, recombinant viral vaccines by virtue of their expression of foreign gene products in vivo meet the broad definition. Given the high level of immunogenicity of vaccinia and other poxvirus and the ease in generating recombinants noted above, poxvirus recombinants have been used extensively as vaccines for infectious organisms and more recently tumors. Preclinical studies using a variety of tumor transplants in wild-type and antigen-expressing transgenic mice have provided a strong basis for the use of poxvirus vaccines clinically (reviewed in Ref. [13]). Promising preclinical results have led to early stage clinical trials of vaccinia and nonreplicating poxviral vectors encoding defined tumor antigens such as CEA and PSA administered using a single viral vector or combinations of vectors in complex primeboost strategies with and without genes encoding immune active cytokines and/or costimulatory molecules. ${ }^{14-17}$

\section{Cytokine gene transfer studies in antitumor immunity}

The generation of a cell-mediated immune response to tumor or any antigen requires: (a) the presence of an antigen or target on the tumor; (b) the presence of a T-lymphocyte population with specificity for the particular antigen; and (c) a supportive cytokine milieu responsible for the recruitment of appropriate antigen-presenting cells and the modulation of the resultant response. ${ }^{6}$ Numerous studies both preclinical and clinical from a variety of investigators have shown that tumor-specific and/or tumor-associated antigens are present on tumors and that following in vivo and/or in vitro expansion, antigen-specific T-cell populations are demonstrable. Based on these and other findings, a major emphasis in current vaccine design has been placed on attempts to modulate responses by combining cytokine genes and/or genes encoding cell surface costimulatory molecules such as B7.1, LFA-3, and ICAM-1, which are expressed on professional antigen-presenting cell populations and not normally expressed on most tumors, together with genes encoding tumor antigen in single viral or DNA-based constructs. Towards this end, a number of laboratories have stably transfected murine and, more recently, human tumor with a variety of such genes for use as vaccines. ${ }^{18-27}$ In murine studies, such manipulation almost uniformly resulted in rejection of the transfected and coinjected nontransfected tumor. In some cases, mice were shown to generate a measurable systemic antitumor response based on rejection of subsequent challenge with the nontransfected tumor. ${ }^{18,19,28,29}$ In a limited number of cases, "vaccination" with such cells resulted in the elimination or reduced growth of preexisting tumor. ${ }^{22,23,29}$ Whereas these studies have been less than overwhelming in their effects on existing tumors, they do show that localized cytokine/lymphokine production can enhance the generation of tumor-specific immunity. More recently, this approach has been translated to clinical trials in renal carcinoma, prostate cancer, and melanoma. ${ }^{25-27,30-32}$ While, for the most part, there have been limited clinical antitumor responses, studies have shown positive immunologic findings. $25-27,30-32$

While the clinical trials to date using ex vivo transfected autologous tumor have demonstrated intriguing results, the requirement that autologous tumor, based on the need for proper antigen and MHC expression, be available, removed, transfected, cloned, and so forth, severely limits the number of suitable patients. A modified approach using cytokinetransfected HLA-matched allogeneic tumor or transfected autologous fibroblasts is currently under study at a number of centers, which may increase the numbers of potential patients. $^{33-35}$ However, the labor-intensive nature of such approaches continues to be a limitation.

\section{In situ cytokine gene transfer to enhance antitumor immunity}

Given the limitations and the lack of clear successes resulting from the approaches outlined above, we developed a strategy of directly inserting the desired cytokine gene or geneencoding costimulatory antigens into the tumor utilizing vaccinia virus recombinants. Injection of the virus intralesionally or intravesically in the case of bladder cancer would result in the infection of the tumor cells and, subsequently, the secretion of biologically active cytokines and/or the cell surface expression of costimulatory molecules. Supported by the preclinical tumor transfection studies described above, it is our hypothesis that production of proimmune cytokines locally at the tumor site in this way would enhance the generation of systemic tumor-specific immunity and resultant tumor destruction. We chose vaccinia virus vectors for our initial studies for a number of reasons. As noted above, vaccinia replicates solely in the cytoplasm, infects a variety of cell types with high efficiency, has the capacity to express several encoded genes in the same vector, and has 
been used in countless millions of individuals to eradicate smallpox.

\section{Tumor transfection by vaccinia recombinants}

The overall hypothesis behind our studies is that by modulating the immune milieu at the local tumor site, and thus recruiting antigen-presenting and effector cell populations, it will be possible to engender a systemic tumorspecific immune response. The result would be to eliminate both localized and disseminated tumor. We pursued both preclinical and clinical studies to determine the feasibility of the use of recombinant vaccinia as a vector for in situ transfection with the result that highly supportive data have been generated, which enhances our enthusiasm for the approach.

Prior to developing recombinant vaccinia for our clinical studies, it was necessary to demonstrate that vaccinia virus recombinants were capable of transfecting murine and human tumor cells. A panel of cell lines including the murine melanoma B16, bladder tumors MBT2 and MB49, ${ }^{36,37}$ as well as human melanoma lines produced from our patients, ${ }^{38,39}$ bladder (T24), and prostate carcinoma (LNCAP, PC3) cell lines ${ }^{40}$ were examined for their ability to be infected/transfected with vaccinia recombinants. Cell lines were exposed in vitro to vaccinia virus recombinants encoding the genes for influenza hemagglutinin and nuclear protein antigens, which allow us to stain for productively infected/transfected cells in vitro. Without exception, all cell lines tested were highly susceptible to infection/transfection at a multiplicity of infection (MOI) of 10:1 PFU:cell. ${ }^{37,41}$

To determine if recombinant vaccinia were able to infect/ transfect tumor in vivo, vaccinia recombinants containing reporter constructs (HA, NP, or the lacZ gene) were injected intralesionally into murine B16 melanoma lesions or instilled through urethral catheters into the bladders of C57BL/6 mice bearing the MB49 tumor, ${ }^{36,37,42}$ with results demonstrating significant infection/transfection in all systems tested. Given the immunogenicity of vaccinia and the possibility that immunity to the virus would prevent infection/transfection following in vivo administration, we examined tumor infection/transfection in vaccinia immune recipients and demonstrated significant activity in vacciniaimmune mice m,41 $^{37,4 l o w i n g ~ i n t r a l e s i o n a l ~ o r ~ i n t r a v e s i c a l ~}$ administration. Thus, systemic immunity to vaccinia, which would be expected to be present in adult patients and following initial vaccinia treatments, does not prevent in vivo tumor infection/transfection.

\section{Cytokine gene delivery using vaccinia recombinants}

To determine if vaccinia recombinants could be used to transfect tumors with resultant cytokine production, we established a panel of vaccinia recombinants expressing murine IL-4, IL-5, IFN $\delta$, and GMCSF. ${ }^{42,43}$ At the time at which these were being produced, a report by Ramshaw et al demonstrated cytokine production by such recombinants. They subsequently demonstrated that the resultant production of cytokines at the viral infection/immunization site had profound effects on the resultant antiviral immunity and viral clearance. Cytokines such as IL-2 and IL-12 demonstrated enhanced viral clearance and immunity, IFN $-\gamma$ and TNF showed significant direct antiviral activity, whereas IL-4 significantly inhibited viral clearance and immunity. ${ }^{44}$ Our ELISA and functional cytokine analyses demonstrated that the vaccinia-recombinant-infected tumor cells produce significant levels of cytokine protein ${ }^{43}$ and, using vaccinia-specific primers designed in our laboratory which allow

Table 1 Intratumoral injection of the vaccinia-GMCSF recombinant virus in patients with malignant melanoma

\begin{tabular}{|c|c|c|c|c|c|c|c|c|c|}
\hline Patient & Age/sex & Metastases & Prior treatment & $\begin{array}{c}\text { Total } \\
\text { lesions/ } \\
\text { sessions }\end{array}$ & $\begin{array}{l}\text { Total dose } \\
\left(\times 10^{7} \text { PFU }\right)\end{array}$ & $\begin{array}{c}\text { Anti vaccinia } \\
\text { titer }\end{array}$ & $\begin{array}{c}\text { Anti } \\
\beta \text {-gal } \\
\text { titer }\end{array}$ & $\begin{array}{l}\text { Regressing } \\
\text { injected } \\
\text { lesions }\end{array}$ & $\begin{array}{c}\text { Regressing } \\
\text { uninjected } \\
\text { lesions: regional }\end{array}$ \\
\hline 1 & $81 / F$ & Dermal, LN & Radiation & $12 / 26$ & 74.73 & 180 & 220 & $\begin{array}{l}\text { Dermal - yes; } \\
\text { Nodal - no }\end{array}$ & Dermal - yes \\
\hline 2 & $68 / M$ & $\begin{array}{l}\text { SC, LN, } \\
\text { lung }\end{array}$ & BCDT, Taxol & $12 / 27$ & 82.0 & 80 & 30 & $\begin{array}{l}\text { Subcutaneous - no; } \\
\text { Nodal }- \text { no }\end{array}$ & Nodal - no \\
\hline 3 & $32 / F$ & $\begin{array}{l}\text { Dermal } \\
\text { Breast }\end{array}$ & $\begin{array}{l}\text { Radiation, } \\
\text { BCDT, } \\
\text { DCV+IL2+ } \\
\text { IFN, Taxol }\end{array}$ & $\begin{array}{l}\text { Dermal } \\
45 / 56 \\
\text { Breast 2/8 }\end{array}$ & $\begin{array}{r}207.3 \\
20.5\end{array}$ & 225 & 100 & $\begin{array}{l}\text { Dermal - yes } \\
\text { Breast - no }\end{array}$ & Dermal - yes \\
\hline 4 & $61 / F$ & $\begin{array}{l}\text { Dermal, SC } \\
\text { LN, lung }\end{array}$ & BCDT, IFN & $9 / 11$ & 49 & 400 & 200 & $\begin{array}{l}\text { Dermal - yes; } \\
\text { Subcutaneous - yes }\end{array}$ & Nodal - no \\
\hline 5 & $71 / F$ & Dermal, LN & $\begin{array}{l}\text { Limb } \\
\text { perfusion } \\
\text { GP100, } \\
\text { MARTI, } \\
\text { IL-12, } \\
\text { BCDT }\end{array}$ & $17 / 12$ & 47 & 200 & 75 & Dermal - yes & Dermal - yes \\
\hline 6 & $67 / M$ & $\begin{array}{l}\text { SC, LN, } \\
\text { lung }\end{array}$ & BCDT & $13 / 13$ & 64 & $>300$ & 80 & Subcutaneous - no & $\begin{array}{l}\text { Subcutaneous - no; } \\
\text { Nodal }- \text { no }\end{array}$ \\
\hline 7 & $75 / M$ & Dermal & None & $13 / 11$ & 47.1 & 100 & 50 & Dermal - yes & Dermal - yes \\
\hline
\end{tabular}

PFU, plaque-forming units; LN, Iymph node; SC, subcutaneous; IL, interleukin; BCDT, BCNU + cicplatin + DTIC+ tamoxifen; IFN, interferon; DCV, DTIC + cisplatin + vinblastine. 
the elucidation of encoded cytokine mRNA in vivo, demonstrated prolonged cytokine gene expression in vivo following intralesional injection

\section{Intralesional vaccinia vector in patients with melanoma}

As a prelude to studying the effects of intralesional recombinant vaccinia in human melanoma, we obtained an IND from the FDA to inject the Wyeth strain of vaccinia (the vaccine used in the US for smallpox immunization and our nonrecombinant parent) intralesionally in patients with recurrent superficial melanoma. ${ }^{39,45}$ Five patients with histologically documented, surgically incurable melanoma with at least one dermal, subcutaneous, or lymph node metastasis that was evaluable for local response and accessible for injection were enrolled. All patients were immunocompetent as demonstrated by one or more positive cutaneous delayed-type hypersensitivity reactions to recall microbial antigens or to dinitrofluorobenzene after sensitization. Following the demonstration of systemic immunity to vaccinia through an intradermal administration of vaccine to the patients, increasing doses of vaccinia were injected intratumorally. Patients were treated twice weekly with increasing doses of intralesionally injected virus. The number of treatments and total dose varied from a low of four treatments for a total of $8 \times 10^{6}$ PFU to a high of 19 treatments for a total of $12.85 \times 10^{7}$ PFU. Four of five patients developed high titers of anti-vaccinia antibody within 14-21 days. (For a detailed description of treatment and response of each patient see, Ref. [46].) Antitumor activity varied among the patients, with one patient exhibiting no regression of treated nor untreated lesions, three patients had partial but brief ( $<1$ month $)$ regression of treated lesions with the onset of progression coinciding with the failure to maintain erythema and induration with repeated injections of
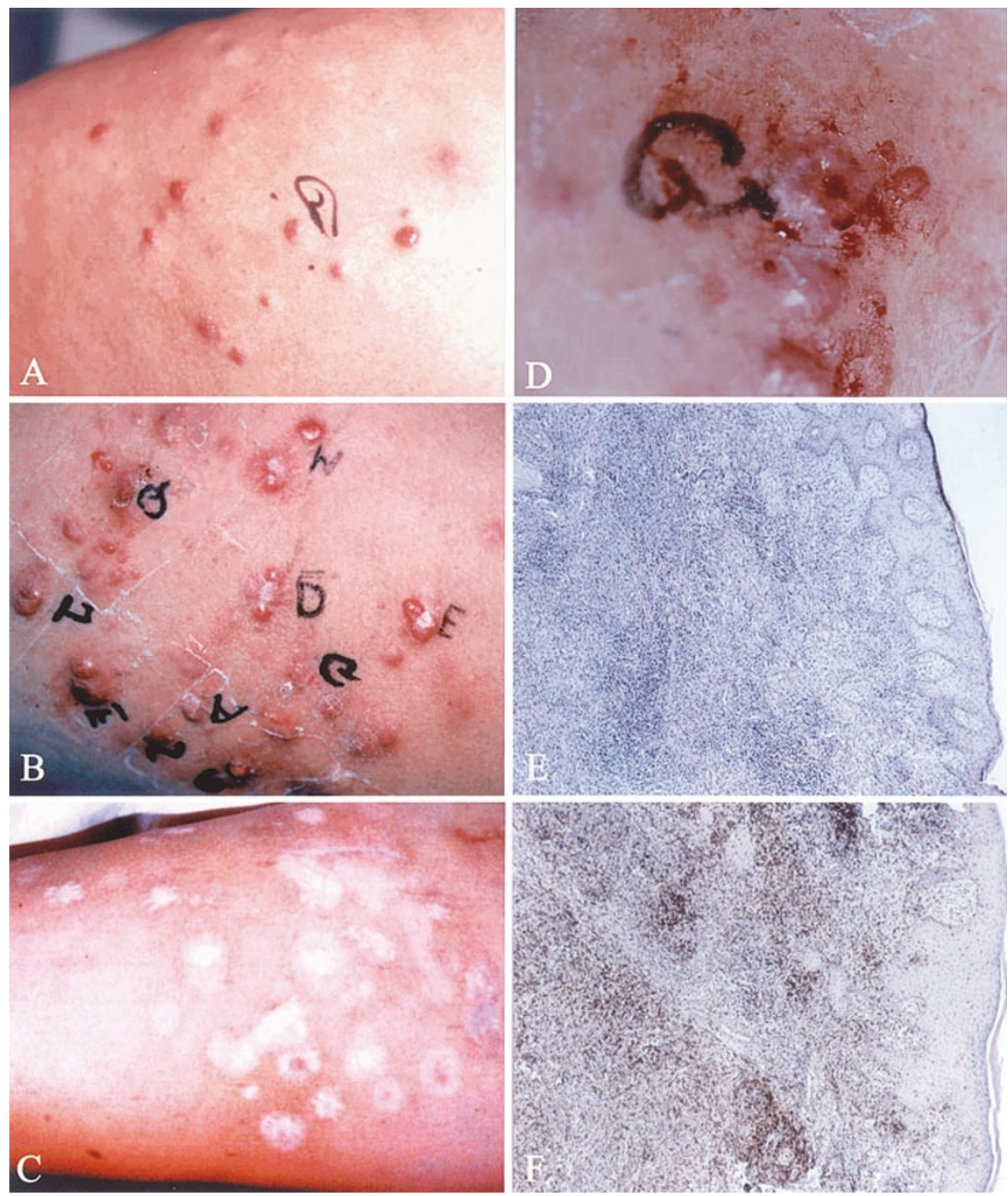

Figure 1 Resolution of dermal metastases following intralesional injection of recombinant vaccinia-GMCSF. Patient 3, a 32 -year - old female with extensive dermal metastases of the left thigh before treatment (A), on day 81 (B), and on day 600, 150 days following cessation of treatment (C). Regression was accompanied by gross (D) and histologic (E) evidence of inflammation including significant $T$-cell $\left(C D 3^{+}\right)$involvement $(F)$ (reprinted with permission from Cancer Gene Ther 1999;6:409-422). 
virus. The fifth patient sustained clinically complete remission of a large exophytic mass that was treated repeatedly as well as a smaller untreated lesion in close proximity. The size of the lesion in this latter patient allowed serial biopsies to be taken to assess viral gene function over time. In this patient, $10^{6}$ PFU was injected into four sites in a 3 -cm superficial melanoma lesion. The lesion was biopsied at 6 hours and 4 days following administration. The biopsies were processed as frozen sections and stained using the monoclonal antibody TW2-3, which is specific for an early viral protein product of the EL3 gene present at sites of viral replication. ${ }^{39}$ To determine if increasing immunity to vaccinia induced by multiple treatments would block productive infection/transfection, additional biopsies were similarly analyzed over the course of therapy in this patient who received 19 biweekly injections of as high as $10^{7} \mathrm{PFU}$ of virus (total cumulative dose of $12.85 \times 10^{7} \mathrm{PFU}$ ). Whereas the duration of expression was diminished with increasing immunity as measured by antiviral antibody titer, productive infection was seen throughout the treatment course. ${ }^{39,45}$ It should be noted that minimal systemic side effects were seen in the trial. These findings demonstrate, as did our murine studies above, that vaccinia recombinants are able to infect/ transfect tumor in vivo following intralesional injection even in the face of systemic immunity to the virus. We concluded from these studies that systemic immunity to the virus acts to protect the patients from toxicity while not preventing local gene expression. Our demonstration of sustained infection in virus-immune individuals strongly supports our approach in demonstrating that infection/transfection using cytokine gene-encoding vaccinia should result in cytokine production for a prolonged period.

\section{Intralesional vaccinia-GMCSF recombinant in patients with melanoma}

Having been satisfied that the vaccinia vector met our requirements of safety and efficacy, we carried out a Phase I trial of intralesional vaccinia-GMCSF in patients with therapy-refractory recurrent melanoma. The recombinant virus was produced in our laboratories using the Wyeth vaccine strain of vaccinia obtained from the CDC, which was also used in our vector alone trial above. The full-length cDNA for human GMCSF was obtained from the American Type Culture Collection (Manassas, VA) (pCSF - 1, no.
39754 ) and cloned behind the pSC65 Synthetic Early/Late Promoter provided by Dr Bernard Moss (NIAID). The $\beta$-gal gene was included as a reporter gene cloned behind the p7.5 Early/Late vaccinia promoter. (For a complete description of the viral construct and production, see Ref. [47].) Clinical grade virus was produced under GMP conditions, allowing us to obtain an IND from the FDA (BB-IND-6486). All patients were required to have accessible dermal and/or subcutaneous disease with a number also having visceral disease. Following the demonstration of immune competence - important given the replicative nature of the vector - patients receive twice weekly intralesional injections of the recombinant with dose escalation within each patient. Patients were initially treated for a 4 -week induction period (eight treatments). Those patients who continued to manifest clinical indications of local infectivity were maintained on treatment until maximal benefit or tumor progression. Table 1 summarizes the results seen in the first seven patients who are described in detail in Ref. [47]. At the highest doses, patients received $2 \times 10^{7} \mathrm{PFU}$ per lesion with the injection of multiple lesions resulting in as high as $8 \times 10^{7}$ per session. For comparison, vaccinia was used as a smallpox immunization at a scarification dose of $2.5 \times 10^{5}$. The two patients with the largest tumor burdens failed to respond even at treatment sites. Three patients had mixed responses, with regression of treated and untreated dermal metastases and progression of disease elsewhere. One patient had a partial response, with regression of injected and uninjected regional dermal metastases. Residual melanoma was excised, rendering the patient disease-free. One patient with only dermal metastases confined to the scalp achieved a complete remission.

Figure 1 demonstrates the complete resolution of dermal metastases seen in patient 3 of the study following treatment and that tumor eradication was accompanied by recruitment of large numbers of $\mathrm{CD}^{+} \mathrm{T}$ cells (both CD4 and CD8 phenotype, not shown) into injected lesions. Figure 2 demonstrates the regression of a noninjected lesion outside of the lymphatic drainage area from the injected site (below the knee on the back of the calf) associated with significant $\mathrm{CD}^{+} \mathrm{T}$-cell infiltration. It was the regression of uninjected lesions in four of seven patients that we have taken as evidence of the induction of tumor-specific immunity. Laboratory studies demonstrated that patients developed high levels of immunity to both vaccinia and the included $\beta$-galactosidase gene product. ${ }^{47}$ We also confirmed that, in
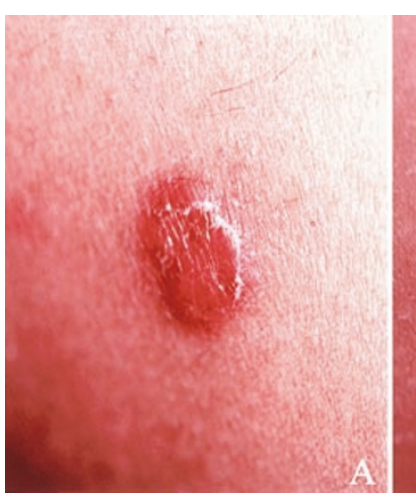

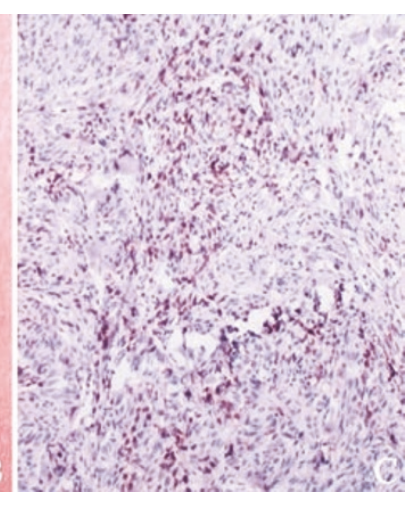

Figure 2 Regression of uninjected lesions is accompanied by $\mathrm{T}$-cell infiltration. A representative uninjected distant regressing lesion prior to (A) and following patient treatment (B) demonstrated T-cell (CD8) infiltration (C) (reprinted with permission from Cancer Gene Ther 1999;6:409-422). 


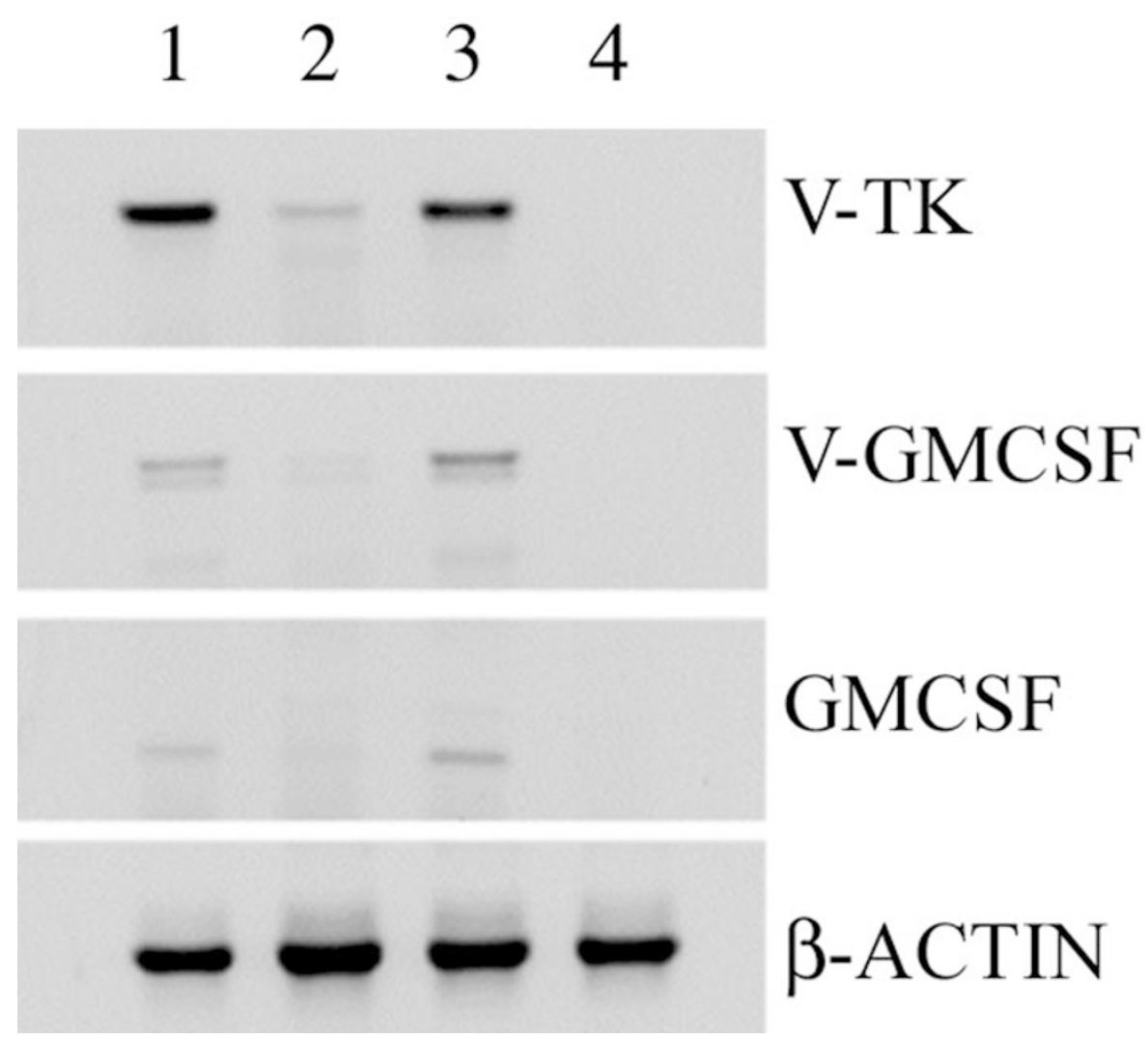

Figure 3 Expression of vaccinia-encoded genes following intralesional injection. RT-PCR of mRNA from melanoma biopsies for vaccinia thymidine kinase (V-TK); vaccinia- encoded human GMCSF (V-GMCSF); human GMCSF (GMCSF); and biopsies from injected (lanes 1-3) and an uninjected lesions. Lane 1: Biopsy 18 hours following the last of a series of multiple injections; lanes 2 and 3: biopsies 18 hours following a single injection; lane 4: uninjected lesion. All biopsies were taken from patient 3 at week 31 (reprinted with permission from $J$ Clin Invest 2000;105:1031).

the face of maximal antibody titers, we continued to be successful at achieving recombinant gene expression as measured both as vaccinia-encoded GMCSF (V-GMCSF) (RT-PCR using primers designed to specifically identify viral encoded GMCSF) and viral thymidine kinase (V-TK) gene mRNA expression (Fig 3).

\section{Intravesical vaccinia in patients with bladder cancer}

As a first step to our planned expansion of this strategy to the localized treatment of bladder cancer (our preclinical data demonstrated significant infection/transfection of the orthotopically growing murine bladder tumor MB49 following intravesical administration of recombinant vaccinia ${ }^{37}$ ), we have completed a Phase I study of intravesical vaccinia vector in patients with advanced transitional cell carcinoma. As with our Phase I study of vaccinia vector alone in melanoma, ${ }^{39}$ we used the vaccinia vector in a dose escalation study, with each patient receiving three intravesical doses over a 2-week period. Given safety concerns, this study focused on patients with invasive transitional cell carcinoma scheduled for cystectomy with the cystectomy scheduled for the day following the third dose. Table 2 summarizes patient characteristics, doses employed, and toxicity. As noted in our prior clinical trials, patients developed high titers of antivaccinia antibody, although maximal titers were meas- ured after cystectomy given the shortened course of therapy (not shown). Also as noted above, treatment was associated with a significant recruitment of activated $\mathrm{T}$ lymphocytes. Figure 4 demonstrates recruitment of activated $\mathrm{CD}^{+}{ }^{+} \mathrm{T}$ lymphocytes as well as dendritic cells that we feel will enhance prospects for the induction of immunity to tumor.

\section{Completed and ongoing clinical trials of localized immune gene transfer}

Subsequent to our preclinical and clinical studies outlined above, there have been a number of clinical trials carried out or in the planning stage that utilize the above strategy. Table 3 outlines studies using vaccinia as well as related replicating ${ }^{39,47-52}$ and nonreplicating poxvirus vectors

Table 2 Intravesical vaccinia vector prior to cystectomy in patients with muscle-invasive bladder cancer

\begin{tabular}{lclll}
\hline Patient & Age/sex & $\begin{array}{c}\text { Dose/treatment } \\
\left(\times 10^{6} \mathrm{PFU}\right)\end{array}$ & \multicolumn{1}{c}{ Toxicity } & $\begin{array}{c}\text { Bladder } \\
\text { inflammation }\end{array}$ \\
\hline 1 & $57 / \mathrm{F}$ & $1,5,10$ & Mild dysuria & Slight \\
2 & $36 / \mathrm{M}$ & $10,25,100$ & Mild dysuria & Significant \\
3 & $64 / \mathrm{M}$ & $25,100,100$ & Mild dysuria & Significant \\
4 & $52 / \mathrm{M}$ & $25,100,100$ & Mild dysuria & Significant \\
\hline
\end{tabular}



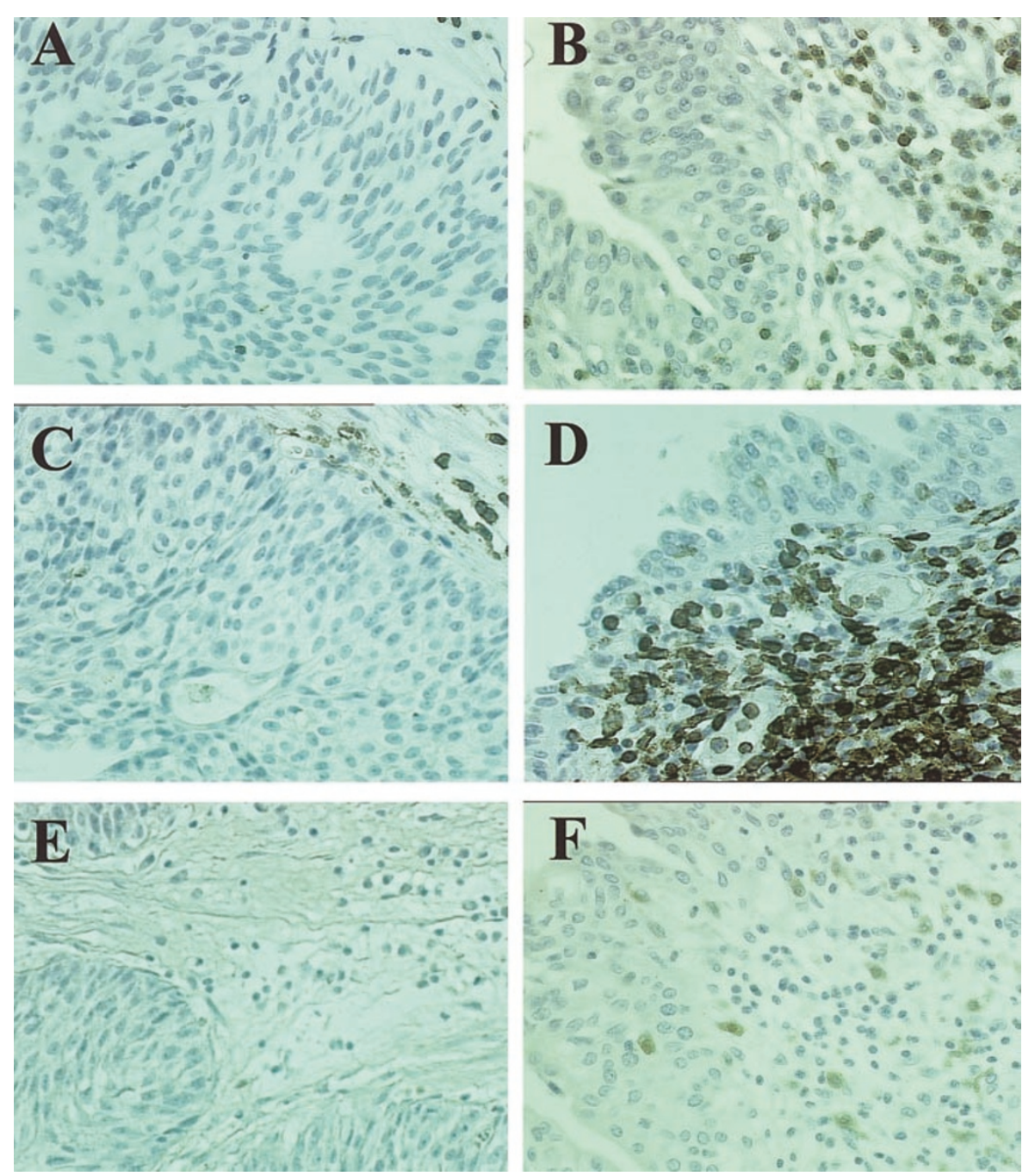

Figure 4 Recruitment of activated T lymphocytes and dendritic cells to the bladder wall following intravesical vaccinia instillation. Immunohistochemical staining of bladder from patient 2. Pretreatment biopsies (A,C,E) and posttreatment cystectomy sections (B,D,F) were stained for CD3 (A,B), CD45RO (C,D), and Factor XIIla (dendritic cells) (E,F) (reprinted with permission from J Clin Invest 2000;105:1031).

encoding cytokines such as $\mathrm{GMCSF}^{47}$ and IL-2 $2^{49,50}$ with and without cell surface immune costimulatory molecules such as B7.1 alone or in combination with LFA-3 and ICAM-1 (TRICOM) $)^{51,52}$ and shown above in preclinical studies to enhance the interaction of antigen-presenting cells with antigen-specific $\mathrm{T}$ lymphocytes used in a variety of tumors. Whereas antitumor responses have been varied in the studies completed to date, demonstration of significant

Table 3 Clinical trials of localized immune gene transfer to solid tumors

\begin{tabular}{|c|c|c|c|c|c|c|}
\hline Investigators & Tumor & Vector & Administration & Patients & Status & References \\
\hline $\begin{array}{l}\text { Mastrangelo, } \\
\text { Lattime }\end{array}$ & Melanoma & Vaccinia & Intralesional & 5 & Complete & {$[38,39]$} \\
\hline $\begin{array}{l}\text { Mastrangelo, } \\
\text { Lattime }\end{array}$ & Melanoma & Vaccinia-GMCSF & Intralesional & 7 & Complete & [47] \\
\hline $\begin{array}{l}\text { Gomella, } \\
\text { Lattime }\end{array}$ & Bladder & Vaccinia & Intravesical & 3 & Complete & {$[48]$} \\
\hline $\begin{array}{l}\text { Robinson, } \\
\text { Scott }\end{array}$ & Mesothelioma & Vaccinia-IL-2 & Intralesional & 6 & Complete & {$[49,50]$} \\
\hline Kaufman & Melanoma & Vaccinia-B7.1 & Intralesional & $6-12$ & Accruing & [51] \\
\hline Kaufman & Melanoma & Vaccinia-TRICOM & Intralesional & $6-12$ & Accruing & [52] \\
\hline $\begin{array}{l}\text { Wojtowicz, } \\
\text { Lattime }\end{array}$ & Bladder & $\begin{array}{l}\text { Fowlpox-GMCSF } \\
\pm \text { Fowlpox TRICOM }\end{array}$ & Intravesical & $24-42$ & $\begin{array}{l}\text { Accrual } \\
\text { beginning } \\
\text { Fall } 2002\end{array}$ & N/A \\
\hline
\end{tabular}


tumor transfection following repeated injections where neutralizing antibodies are found is uniformly seen supporting the use of poxvirus as vectors for sustained production of immune-enhancing cytokines and cell surface molecules in vivo.

\section{Conclusions}

In summary, we have discussed an approach to immunologically based gene therapy logically designed from the requirements to generate a productive cellular immune response. As outlined in this review, numerous strategies have been hypothesized and tested in both preclinical and clinical settings with this goal as an endpoint.

It is our hypothesis that in situ tumor transfection with cytokine genes will provide a logical extension of the vaccine strategies that have been previously studied. By incorporating genes selected based on their known contribution to the generation of systemic immune responses, we anticipate the ability to optimize the generation of an antitumor response. In addition to this logical in vivo vaccine design, this methodology will allow the generation of a single reagent in a bottle that will be of use in any tumor type provided that it is accessible to injection. This will preclude the need to have sufficient autologous tumor for harvest and subsequent vaccine production and will overcome the significant limitation of the in vitro transfectants for tumor transfection and selection in the laboratory. As noted above, the use of the patients' own tumor as a source of antigens in our system optimizes the generation of a $\mathrm{T}$-cell response and has significant advantages over allogeneic vaccine strategies that rely on shared antigens restricted by common MHC antigens.

\section{Acknowledgments}

Supported by ACS Grants IM - 742 and EDT-78842; USPHS Grants CA-42908, CA-55322, CA-69253, CA-74543; and the Nat Pincus Trust.

\section{References}

1. Lattime EC, Gerson SL. Gene Therapy of Cancer: Translational Approaches from Preclinical Studies to Clinical Implementation. 2nd ed. San Diego: Academic Press; 2002:534.

2. Thomas L. In: Lawrence HS, ed. Cellular and Humoral Aspects of the Hypersensitive States. New York: Hoeber-Harper; 1959: 529-532.

3. Burnet FM. The concept of immunological surveillance. Prog Exp Tumor Res. 1970;13:1.

4. Bodurtha AJ, Berkelhammer J, Kim YH, Laucius JF, Mastrangelo MJ. A clinical, histologic, and immunologic study of a case of metastatic malignant melanoma undergoing spontaneous remission. Cancer. 1976;37:735-742.

5. Spontaneous Remission: An Annotated Bibliography. Sausalito, CA: Institute of Noetic Sciences; 1993:1-710.

6. Ostrand-Rosenberg S, Clements VK, Dissanayake S, Gilbert M, Pulaski BA, Qi L. Immunologic targets for the gene therapy of cancer. In: Lattime EC, Gerson SL, eds. Gene Therapy of Cancer: Translational Approaches from Preclinical Studies to Clinical Implementation. 2nd ed. San Diego: Academic Press; 2002:128-144.

7. Bornstein RS, Mastrangelo MJ, Sulit H, et al. Immunotherapy of melanoma with intralesional BCG. Natl Cancer Inst Monogr. 1973;39:213-220.

8. Laucius JF, Bodurtha AJ, Mastrangelo MJ, Creech RH. Bacillus Calmette-Guerin in the treatment of neoplastic disease. J Reticuloendothel Soc. 1974;16:347-373.

9. Lamm DL, Thor DE, Harris SC, Reyna JA, Stogdill VD, Radwin HM. Bacillus Calmette-Guerin immunotherapy of superficial bladder cancer. J Urol. 1980;124:38-42.

10. Mastrangelo MJ, Maguire HCJ, Lattime EC, Berd D. Whole cell vaccines. In: DaVita VT, Hellman S, Rosenberg SA, eds. Biological Therapy of Cancer. 2nd ed. Philadelphia: Lippincott; 1995:648-658.

11. Mastrangelo MJ, Sato T, Lattime EC, Maguire HC Jr, Berd D. Cellular vaccine therapies for cancer. In: Foon KA, Muss HB, eds. Biological and Hormonal Therapies of Cancer. Boston: Kluwer Academic Publishing; 1998:35-50.

12. Hu X, Chakraborty NG, Sporn JR, Kurtzman SH, Ergin MT, Mukherji B. Enhancement of cytolytic T lymphocyte precursor frequency in melanoma patients following immunization with MAGE-1 peptide loaded antigen presenting cell-based vaccine. Cancer Res. 1996;56:2479-2483.

13. Schlom J, Tsang K-Y, Kantor J, et al. Strategies in the development of recombinant vaccines for colon cancer. Semin Oncol. 1999;26:672-682.

14. Marshall JL, Hoyer RJ, Toomey MA, et al. Phase I study in advanced cancer patients of a diversified prime-and-boost vaccination protocol using recombinant vaccinia virus and recombinant nonreplicating avipox virus to elicit anti-carcinoembryonic antigen immune responses. J Clin Oncol. 2000; 18:3964-3973.

15. Horig H, Lee DS, Conkright W, et al. Phase I clinical trial of a recombinant canarypoxvirus (ALVAC) vaccine expressing human carcinoembryonic antigen and the B7.1 co-stimulatory molecule. Cancer Immunol Immunother. 2000;49:504-514.

16. Eder JP, Kantoff PW, Roper K, et al. A phase I trial of a recombinant vaccinia virus expressing prostate-specific antigen in advanced prostate cancer. Clin Cancer Res. 2000;6:16321638.

17. Sanda MG, Smith DC, Charles LG, et al. Recombinant vaccinia-PSA (PROSTVAC) can induce a prostate-specific immune response in androgen-modulated human prostate cancer. Urology. 1999;53:260-266.

18. Fearon ER, Pardoll DM, Itaya T, et al. Interleukin- 2 production by tumor cells bypasses $\mathrm{T}$ helper function in the generation of an antitumor response. Cell. 1990;60:397-403.

19. Asher AL, Mulé JJ, Kasid A, et al. Murine tumor cells transduced with the gene for tumor necrosis factor- $\alpha$ : evidence for paracrine immune effects of tumor necrosis factor against tumors. J Immunol. 1991;146:3227-3234.

20. Watanabe Y, Kuribayashi K, Miyatake J, et al. Exogenous expression of mouse interferon-gamma cDNA in mouse neuroblastoma $\mathrm{C} 1300$ cells results in reduced tumorigenicity by augmented anti-tumor immunity. Proc Natl Acad Sci USA. 1989;86:9456-9460.

21. Tepper RI, Pattengale PK, Leder P. Murine interleukin-4 displays potent anti-tumor activity in vivo. Cell. 1989;57:503512.

22. Connor J, Bannerji R, Saito S, Heston W, Fair W, Gilboa E. Regression of bladder tumors in mice treated with interleukin 2 gene-modified tumor cells. J Exp Med. 1993;177:11271134. 
23. Saito S, Bannerji R, Gansbacher B, et al. Immunotherapy of bladder cancer with cytokine gene-modified tumor vaccines. Cancer Res. 1994;54:3516-3520.

24. Dranoff G, Jaffee E, Lazenby A. Vaccination with irradiated tumor cells engineered to secrete murine granulocyte-macrophage colony stimulating factor stimulates potent, specific, and long lasting anti-tumor immunity. Proc Natl Acad Sci USA. 1993;90:3539-3543.

25. Simons JW, Mikhak B, Chang JF, et al. Induction of immunity to prostate cancer antigens: results of a clinical trial of vaccination with irradiated autologous prostate tumor cells engineered to secrete granulocyte-macrophage colony-stimulating factor using ex vivo gene transfer. Cancer Res. 1999;59: 5160-5168.

26. Simons JW, Jaffee EM, Weber CE, et al. Bioactivity of autologous irradiated renal cell carcinoma vaccines generated by $e x$ vivo granulocyte-macrophage colony-stimulating factor gene transfer. Cancer Res. 1997;57:1537-1546.

27. Nelson WG, Simons JW, Mikhak B, et al. Cancer cells engineered to secrete granulocyte-macrophage colony - stimulating factor using ex vivo gene transfer as vaccines for the treatment of genitourinary malignancies. Cancer Chemother Pharmacol. 2000;46:S67-S72.

28. Perussia B, Chan SH, D'Andrea A, et al. Natural killer cell stimulatory factor or interleukin-12 has differential effects on the proliferation of TCR $\alpha \beta+, \mathrm{TCR} \tau \delta+\mathrm{T}$ lymphocytes and NK cells. J Immunol. 1992;149:3495-3502.

29. Golumbek PT, Lazenby AJ, Levitsky HI, et al. Treatment of established renal cancer by tumor cells engineered to secrete interleukin-4. Science. 1991;254:713-716.

30. Kusumoto M, Umeda S, Ikubo A, et al. Phase 1 clinical trial of irradiated autologous melanoma cells adenovirally transduced with human GM-CSF gene. Cancer Immunol Immunother. 2001;50:373-381.

31. Chang AE, Li Q, Bishop DK, Normolle DP, Redman BD, Nickoloff BJ. Immunogenetic therapy of human melanoma utilizing autologous tumor cells transduced to secrete granulocyte-macrophage colony-stimulating factor. Hum Gene Ther. 2000;11:839-850.

32. Soiffer R, Lynch T, Mihm M, et al. Vaccination with irradiated autologous melanoma cells engineered to secrete human granulocyte-macrophage colony-stimulating factor generates potent antitumor immunity in patients with metastatic melanoma. Proc Natl Acad Sci USA. 1998;95:13141-13146.

33. Tahara H, Zeh HJ, Storkus WJ, et al. Fibroblasts genetically engineered to secrete interleukin 12 can suppress tumor growth and induce antitumor immunity to a murine melanoma in-vivo. Cancer Res. 1994;54:182-189.

34. Lotze MT, Rubin JT, Carty S, et al. Gene therapy of cancer: a pilot study of IL-4-gene-modified fibroblasts admixed with autologous tumor to elicit an immune response. Hum Gene Ther. 1994;5:41-55.

35. Kang WK, Park C, Yoon HL, et al. Interleukin 12 gene therapy of cancer by peritumoral injection of transduced autologous fibroblasts: outcome of a phase I study. Hum Gene Ther. 2001;12:671-684.

36. Lee SS, Eisenlohr LC, McCue PA, Mastrangelo MJ, Lattime EC. Intravesical gene therapy: vaccinia virus recombinants transfect murine bladder tumors and urothelium. Proc Am Assoc Cancer Res. 1993;34:337.
37. Lee SS, Eisenlohr LC, McCue PA, Mastrangelo MJ, Lattime EC. Intravesical gene therapy: in-vivo gene transfer using vaccinia vectors. Cancer Res. 1994;54:3325-3328.

38. Lattime EC, Maguire HCJ, McCue PA, et al. Infection of human melanoma cells by intratumoral vaccinia. $J$ Invest Dermatol. 1994;102:568.

39. Mastrangelo MJ, Maguire HC Jr, McCue PA. A pilot study demonstrating the feasibility of using intratumoral vaccinia injections as a vector for gene transfer. Vaccine Res. 1995;4: $55-69$.

40. Gomella LG, Mastrangelo MJ, Eisenlohr LC, McCue PA, Lee SS, Lattime EC. Localized gene therapy for prostate cancer: strategies for intraprostatic cytokine gene transfection using vaccinia virus vectors. J Urol. 1995;153:308A.

41. Lattime E, Eisenlohr L, Gomella L, Mastrangelo M. The use of vaccinia virus vectors for immunotherapy via in-situ tumor transfection. In: Lattime E, Gerson S, eds. Gene Therapy of Cancer: Translational Approaches from Preclinical Studies to Clinical Implementation. San Diego: Academic Press; 1999: $125-137$.

42. Lee SS, Eisenlohr LC, McCue PA, Mastrangelo MJ, Fink E, Lattime EC. In-vivo gene therapy of murine tumors using recombinant vaccinia virus encoding GM-CSF. Proc Am Assoc Cancer Res. 1995;36:248.

43. Lee SS, Eisenlohr LC, McCue PA, Mastrangelo MJ, Lattime EC. Vaccinia virus vector mediated cytokine gene transfer for in vivo tumor immunotherapy. Proc Am Assoc Cancer Res. 1994;35:514.

44. Ramshaw IA, Ramsay AJ, Karupiah G, Rolph MS, Mahalingam S, Ruby JC. Cytokines and immunity to viral infections. Immunol Rev. 1997;159:119-135.

45. Lattime EC, Maguire HCJ, McCue PA, et al. Gene therapy using vaccinia vectors: repeated intratumoral injections result in tumor infection in the presence of anti-vaccinia immunity. Proc Am Soc Clin Oncol. 1994;13:397.

46. Ostrand-Rosenberg S, Pulaski BA, Armstrong TD, Clements VK. Immunotherapy of established tumor with MHC class II and B7.1 cell-based tumor vaccines. Adv Exp Med Biol. 1998;451:259-264.

47. Mastrangelo MJ, Maguire HC Jr, Eisenlohr LC. Intratumoral recombinant GM-CSF-encoding virus as gene therapy in patients with cutaneous melanoma. Cancer Gene Ther. 1999;6:409-422.

48. Gomella LG, Mastrangelo MJ, McCue PA, Maguire HC, Mulholland SG, Lattime EC. Phase I study of intravesical vaccinia virus as a vector for gene therapy of bladder cancer. J Urol. 2001;166:1291-1295.

49. Mukherjee S, Haenel T, Himbeck R, et al. Replicationrestricted vaccinia as a cytokine gene therapy vector in cancer: persistent transgene expression despite antibody generation. Cancer Gene Ther. 2000;7:663-670.

50. Robinson BW, Mukherjee SA, Davidson A, et al. Cytokine gene therapy or infusion as treatment for solid human cancer. J Immunother. 1998;21:211-217.

51. Kaufman HL, Conkright W, Divito J Jr, et al. A phase I trial of intra lesional RV-B7.1 vaccine in the treatment of malignant melanoma. Hum Gene Ther. 2000;11:1065-1082.

52. Kaufman HL, DeRaffele G, Divito J, et al. A phase I trial of intralesional $\mathrm{rV}$-Tricom vaccine in the treatment of malignant melanoma. Hum Gene Ther. 2001;12:1459-1480. 